\title{
SUPERNOVAE AND COSMOLOGY
}

\author{
Norbert Bartel \\ Harvard-Smithsonian Center for Astrophysics \\ 60 Garden Street \\ Cambridge, MA 02138 \\ U.S.A.
}

\begin{abstract}
"Don't believe in any of those methods. The only reliable way of determining extragalactic distances is through supernova investigations."

[Fritz Zwicky - when asked by a student for his opinion on the at that time exclusively used other methods of extragalactic distance estimates. (according to A. Sandage)]
\end{abstract}

\begin{abstract}
I review the presently existing four partly independent methods of determining extragalactic distances through supernova investigations and conclude that, although each of these methods has its difficulties, application of all of them on the same class of objects provides the opportunity of obtaining unique redundancy in determinations of extragalactic distances and Hubble's constant.
\end{abstract}

\section{INTRODUCTION}

The presently existing methods of extragalactic distance determinations through supernova investigations can be divided into two groups. One group of methods allows estimate of the luminosity distance, $d_{L}$, which is given as $d_{L}=(L / 4 \pi \ell)^{1 / 2}$, with $L$ as the absolute luminosity and $\ell$ as the apparent, absorption corrected luminosity. Estimates of $d_{L}$ have been obtained by either determining $L$ empirically (e.g., Cadonau, Sandage, and Tammann 1985) or theoretically (Arnett, Branch, and Wheeler 1985).

The other group of methods allows estimates of the angular diameter distance, $d_{A}$, which is given as $d_{Z}=D / \theta$, with $D$ as the linear diameter of the supernova and $\theta$ as its angular diameter. Estimates of $d_{A}$ have been obtained by using either the Baade-Wesselink method, which was first applied to Type I supernovae (SNeI) by Branch and Patchett (1973) and to Type II supernovae (SNeII) by Kirshner and Kwan (1974), or the VLBI-spectroscopy method (Bartel 1985; Bartel et al. 1985).

In the remainder I discuss each of the four methods in more detail, point out their strengths, underlying assumptions, and possible improvements, and indicate their future prospects. 


\section{EXTRAGALACTIC DISTANCE DETERMINATIONS VIA SUPERNOVA INVESTIGA- TIONS}

\subsection{Luminosity Distance - Empirical Approach}

Since the time when Kowal (1968) demonstrated that SNeI may have a small dispersion of their absolute maximum magnitude, $M(\max )$, SNeI have been seriously considered as candidates for luminosity distance determinations. The $\mathrm{SNel}$ 's high absolute luminosities would make them particularly attractive for most of the methods discussed here since they could probably be observed with the Hubble Space Telescope up to redshifts of $z=0.5$. SNeII, in contrast, are known to have a large dispersion of their $M(\max )$ and are therefore unsuitable for use as luminosity distance indicators. In the empirical approach, $M(\max )$ of an SNI is estimated with a few, more closely located intermediate distance calibrators and then compared with the absorption-corrected apparent maximum magnitude.

The underlying assumptions are that:

i) all SNeI or a distinguishable subclass of them have the same $M(\max )$;

ii) $d_{L}$ is known for at least one SNI or a member of the subclass; and

iii) the apparent maximum magnitude can be corrected with sufficient accuracy for absorption effects.

Observationally SNeI have remarkably homogeneous light curve shapes so that they were thought to have the same $M(\max )$. Recently Cadonau et al. (1985) selected $12 \mathrm{SNeI}$ in assumed-to-be dust-free $\mathrm{E} / \mathrm{SO}$ galaxies and found $M_{B}(\max )=-19.69 \pm 0.13+5 \log \left(H_{0} / 50\right)$. To estimate $H_{0}$ they used two nearby calibrators, SN 1937c in IC4182 and SN 1954a in NGC4214, and made an assumption on the degree of internal absorption. They inferred the calibrator distances by identifying a few red supergiants whose absolute magnitudes were inferred from observations of red supergiants in even closer galaxies. The distances of the latter were estimated through Cepheid observations. Cadonau et al. obtained $M_{B}(\max )=-20.0 \pm 0.4$ and $H_{0}=43_{-7}^{+10} \mathrm{~km} \mathrm{~s}^{-1} \mathrm{Mpc}^{-1}$, the latter value being in agreement with Sandage and Tammann's (1985) value of $H_{0}=50 \pm 7 \mathrm{~km} \mathrm{~s}^{-1} \mathrm{Mpc}^{-1}$. De Vaucouleurs and Corwin (1985) obtained for the local calibrators SN 1572 (Tycho's SN, type not quite certain) and SN 1885 (in M31, SNI with peculiar light curve) a mean of $M_{p g}=-18.55 \pm 0.28$ and a value for $H_{0}$ consistent with de Vaucouleurs and Bollinger's (1979) value of $H_{0}=100 \pm 10 \mathrm{~km} \mathrm{~s}^{-1} \mathrm{Mpc}^{-1}$.

However, it is not clear how reliable the assumptions are. The existence of probably two- to fivefold "underluminous" peculiar SNeI with light curve shapes similar to those of "normal" SNeI (see, e.g., Wheeler and Levreault 1985; Uomoto and Kirshner 1985) could contaminate the sample of $\mathrm{SNeI}$ used to calibrate the extragalactic distance scale. But since the peculiar SNeI have different spectra from the "normal" ones and were found only in spirals, one may hope that a distinguishable subclass of SNeI with the same $M(\max )$ exists.

\subsection{Luminosity Distance - Theoretical Approach}

Arnett, Branch, and Wheeler (1985) attempted to bypass the aforementioned errorprone technique of the step-by-step calibration of the luminosity of $\mathrm{SNeI}$ and computed $L(\max )$ directly from their knowledge of nuclear physics and radiative transfer in the SN shell.

It is thought that an SNI's progenitor is a mass-accreting carbon oxygen white dwarf in a binary system that disrupts completely when its mass approaches the Chandrasekhar mass of $1.4 \mathrm{M}_{\odot}$. The optical luminosity in Arnett et al.'s physical model results from the trapping and thermalization of the $\gamma$-rays and positrons emitted by the decay of ${ }^{56} \mathrm{Ni}$ through ${ }^{56} \mathrm{Co}$ to 
${ }^{56} \mathrm{Fe}$ with the value for $L(\max )$ being a function of the incinerated mass $M_{N i}$ of ${ }^{56} \mathrm{Ni}$. Assuming a blackbody spectrum for SNeI allowed conversion from $L(\max )$ to $M_{B}(\max )$, required for comparison with apparent blue magnitudes of observed SNeI, and thus allowed estimates of distances and $H_{0}$.

The underlying assumptions are that

i) the physical model is correct;

ii) $M_{N i}=0.6_{-0.2}^{+0.8} \mathrm{M}_{\odot}$;

iii) all SNeI have the same $L(\max )$;

iv) all $\mathrm{SNeI}$ at maximum light have continuum blackbody spectra with a temperature of $20000 \mathrm{~K}$; and

v) the apparent luminosity or the apparent magnitude can be corrected for absorption effects with sufficient accuracy.

With these assumptions, Arnett et al. (1985) obtained for SNeI $M_{B}(\max )=-19.5_{-0.9}^{+0.4}$ and $H_{0}=59_{-20}^{+14} \mathrm{~km} \mathrm{~s}^{-1} \mathrm{Mpc}^{-1}$.

However, again, it is not clear how justified their assumptions are. In particular, the existence of underluminous SNeI casts some doubt on the reliability of their estimates of $M_{B}(\max )$ and $H_{0}$. The method needs to predict not only the SN's light curve but also the SN's spectrum in order to be applicable to a distinguishable subclass with the same absolute magnitude.

\subsection{Angular Diameter Distance - Baade-Wesselink Method}

This method was suggested by Baade (1926) for application to Cepheids. The method was first applied to Cepheids by Wesselink (1946a,b; see also 1985) and later to SNeI by Branch and Patchett (1973) and to SNeII by Kirshner and Kwan (1974). Like Arnett et al.'s method, it allows direct distance determinations independent of any intermediate calibrators.

The radial, linear diameter, $D$, is estimated from the $S N$ 's expansion rate, $v_{e x p}$, given by the Doppler broadening of the emission and absorption lines in the SN's spectrum, and the time period, $t$, between the date of explosion and the date of observation. The transverse angular diameter, $\theta$, is estimated from the brightness, $B_{\nu}(T)$ at temperature $T$, and the observed flux density $S_{\nu}$ at frequency $\nu$ so that $d_{L}=D / \theta$ becomes $d_{L}=\left(R_{0}+v_{\exp } t\right)\left(\pi B_{\nu} / S_{\nu}\right)^{+0.5}$ with $R_{0}$ as the (usually ignored) initial radius of the $\mathrm{SN}$ at $t=0$.

The underlying assumptions are that:

i) SNe near maximum light are blackbodies;

ii) SNe are circularly symmetric; and

iii) the observed flux density can be corrected for absorption effects with sufficient accuracy.

Recently Branch et al. (1981) obtained a value for the distance to SN $1979 \mathrm{c}$ in M100, a galaxy in the Virgo cluster, of $23 \pm 3 \mathrm{Mpc}$. If we assume that the distance to $\mathrm{M} 100$ is within $10 \%$ of the distance to the Virgo cluster center, since M100 is within $6^{\circ}$ of this center and has a redshift consistent with this location, and if we use a value for the infall-corrected redshift velocity of the Virgo cluster center of $1250 \pm 150 \mathrm{~km} \mathrm{~s}^{-1}$ (see, e.g., Huchra 1985; Aaronson et al. 1982), we get $H_{0}=54_{-10}^{+13} \mathrm{~km} \mathrm{~s}^{-1} \mathrm{Mpc}^{-1}$.

However, again, it is not clear whether the assumptions are justified. In particular, we would expect deviations from a blackbody spectrum if the opacity at the photosphere were dominated by scattering (Wagoner 1981). Panagia (1985) indeed detected large deviations from a blackbody spectrum in the spectrum of SN 1981b, with a blackbody at $T=15800 \mathrm{~K}$ 
fitting the optical spectrum, and a blackbody at $T=9400 \mathrm{~K}$ fitting the IR and UV spectra. The computation of model SN atmospheres (Hershkowitz, Linder, and Wagoner 1985; Shaviv, Wehrse, and Wagoner 1985) would be useful to free, at least to some extent, distance determinations via this method from assumption i).

\subsection{Angular Diameter Distance - VLBI-Spectroscopy Method}

The VLBI-spectroscopy method (Bartel 1985; Bartel et al. 1985) includes a direct radio interferometric measurement of the angular diameter and the morphology of a SN. When such a measurement is combined with a value of the linear diameter of the SN obtained, like in the Baade-Wesselink method, from the expansion velocity of the SN and the elapsed time since explosion, the SN's distance can be estimated. The advantage of this method over the others is that $\theta$ can be determined directly. However, even the strongest supernovae are relatively weak radio emitters with $S_{5 G H z} \leqslant 10 \mathrm{mJy}$ (Weiler et al. 1985), so that the method is presently restricted to sources not farther away than $\sim 40 \mathrm{Mpc}$.

The underlying assumptions here are that:

i) the diameter of the radiosphere is equal to the diameter of the outer boundary of the SN's shock front, which is given by the maximum width of the SN's emission and absorption lines and the elapsed time since the explosion; and

ii) $\mathrm{SNe}$ are circularly symmetric.

VLBI observations were made of SN 1979c at several epochs after the time of explosion with an array of up to eight telescopes in Europe and the U.S. (Bartel 1985; Bartel et al. 1985). Fig. 1 displays a radio map of SN $1979 \mathrm{c}$ situated in the galaxy M100 in the Virgo cluster.

SN 1979 C IN M100

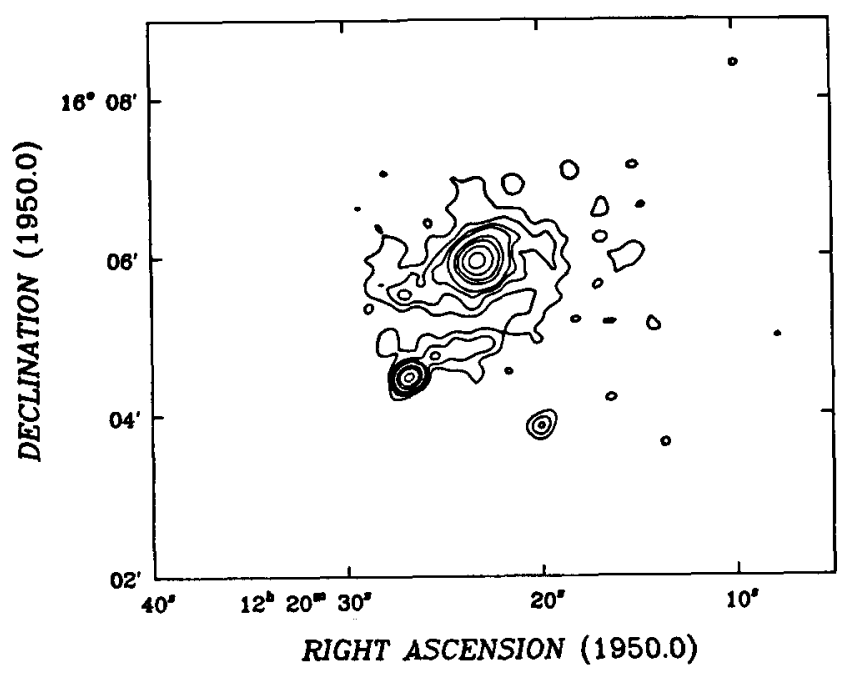

Figure 1. Radio map of the supernova SN 1979c, which is situated at the southern edge of a spiral arm of the galaxy M100.

Use of the most sensitive telescopes and VLBI system enabled determinations of the angular diameter at $5 \mathrm{GHz}$ at three different times and other determinations at other frequen- 
cies. Further observations are planned. The angular diameter determinations at $5 \mathrm{GHz}$ are displayed in Fig. 2 and indicate consistency with uniform expansion $(\theta \propto t)$ of the radiosphere.

\section{SN $1979 c$}

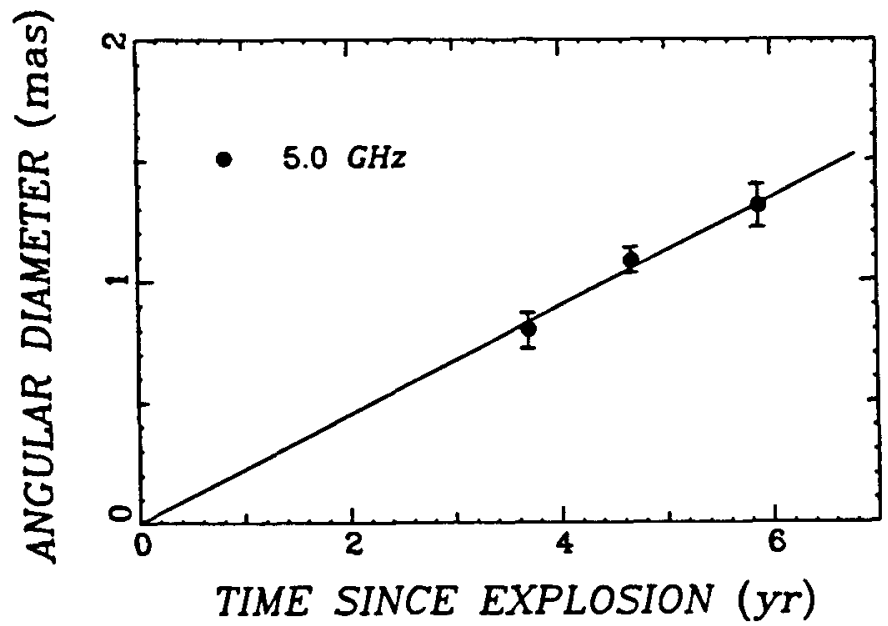

Figure 2. The angular diameter determinations for a shell model. For the extreme models of a ring and a uniform sphere, the ordinate scale has to be multiplied by 0.75 and 1.25 , respectively. The solid line represents uniform expansion. The latest determination is preliminary.

Bartel et al. obtained a value for the distance to SN $1979 \mathrm{c}$ of $19_{-6}^{+8} \mathrm{Mpc}$ and, with the same line of reasoning as mentioned above for the computation of Branch et al.'s value, obtained a value for $H_{0}$ of $65_{-25}^{+35} \mathrm{~km} \mathrm{~s}^{-1} \mathrm{Mpc}^{-1}$. The dominant contribution to the errors is the uncertainty of $\theta$. Since the $\mathrm{SN}$ is still quite compact with respect to the angular resolution obtained with the VLBI array, its morphology has not yet been determined. The value of $\theta$ was computed for an optically thin shell model with an uncertainty of $\pm 25 \%$ given by the predictions from the extreme models of a uniform sphere and a ring (see, e.g., Marscher 1985). Further observations could probably be used to determine the morphology and thus to decrease the uncertainty of $\theta$.

However, again, as for the other methods, it is not clear how justified the assumtions are. In principle, VLBI observations allow determination of the SN's brightness distribution and thus its degree of circular symmetry at the plane on the sky. Bartel et al. have as yet obtained only a coarse bound on any deviation from circular symmetry of the brightness distribution, but more accurate and useful determinations are probably possible. In any event, the degree of circular symmetry in all three dimensions cannot be determined but has to be inferred from plausibility arguments. On the basis of supernova remnant (SNR) observations, they included in their computation of $d_{L}$ and $H_{0}$ an uncertainty of the degree of circular symmetry of the SN's brightness distribution of $\pm 15 \%$.

Also, on the basis of SNR observations and on the basis of considerations of models of the relationship between the radiosphere and the outer boundary of the SN's shock front (see, e.g., Chevalier 1982, 1986; Bandiera, Pacini, and Salvati 1983; Fransson 1986), they included in their computations an uncertainty of assumption i) of also $\pm 15 \%$. More, and more accurate, VLBI measurements of SN 1979c and other supernovae at distances up to $\sim 40 \mathrm{Mpc}$, and modeling of the relationship between the radiosphere and the outer boundary of the SN's shock front could probably result in more accurate values of $d_{L}$ and $H_{0}$. 


\section{CONCLUSIONS}

Investigations of supernovae provide the unique opportunity of obtaining at least partly independent distance determinations with four different methods. Three of these methods allow direct determinations independent of any local distance calibrators. The VLBI-spectroscopy method may (in principle) be the most direct of the four methods since it depends on the least number of assumptions. However, given the present sensitivity of telescopes and VLBI equipment, the method is restricted to supernovae with distances $\leqslant 40 \mathrm{Mpc}$, probably large enough for a more accurate determination of $H_{0}$, but too small for a determination of $q_{0}$. A combination of all four methods may be required to constrain $q_{0}$.

This work was supported by the NSF under grant no. AST-8300796.

\section{REFERENCES}

Aaronson, M., Huchra, J., Mould, J., Schechter, P.L., and Tully, R.B. 1982, Ap. J., 258, 64.

Arnett, W.D., Branch, D., and Wheeler, J.C. 1985, Nature, 314, 337.

Baade, W. 1926, Astr. Nachr., 228, 359.

Bandiera, R., Pacini, F., and Salvati, M. 1983, Astr. Ap., 126, 7.

Bartel, N. 1985, in Supernovae as Distance Indicators, Lecture Notes in Physics, ed. N. Bartel (Springer-Verlag, Berlin), 224, 107.

Bartel, N., Rogers, A.E.E., Shapiro, I.I., Gorenstein, M.V., Gwinn, C.R., Marcaide, J.M., and Weiler, K.W. 1985, Nature, 318, 25.

Branch, D., Falk, S.W., McCall, M.L., Rybski, P., Uomoto, A., and Wills, B.J. 1981, Ap. J., 244, 780.

Branch, D. and Patchett, B. 1973, M. N. R. A. S., 161, 71.

Cadonau, R., Sandage, A., and Tammann, G.A. 1985, in Supernovae as Distance Indicators, Lecture Notes in Physics, ed. N. Bartel (Springer-Verlag, Berlin), 224, 151.

Chevalier, R.A. 1982, Ap. J., 259, 302.

Chevalier, R.A. 1986, this volume.

de Vaucouleurs, G. and Bollinger, G. 1979, Ap. J., 233, 433.

de Vaucouleurs, G. and Corwin, Jr., H.G. 1985, Ap. J., $297,23$.

Fransson, C. 1986, this volume.

Hershkowitz, S., Linder, E., and Wagoner, R.V. 1986, Ap. J., 301, 220.

Huchra, J.P. 1985, in The Virgo Cluster, eds. O.-G. Richter and B. Binggeli, ESO proc. 20, 181.

Kirshner, R.P. and Kwan, J. 1974, Ap. J., 193, 27.

Kowal, C.T. 1968, A. J., 73, 1021.

Marscher, A.P. 1985, in Supernovae as Distance Indicators, Lecture Notes in Physics, ed. N. Bartel (Springer-Verlag, Berlin), 224, 130.

Panagia, N. 1985, in Supernovae as Distance Indicators, Lecture Notes in Physics, ed. N. Bartel (Springer-Verlag, Berlin), 224, 14.

Sandage, A., and Tammann, G.A. 1985, in Supernovae as Distance Indicators, Lecture Notes in Physics, ed. N. Bartel (Springer-Verlag, Berlin), 224, 1.

Shaviv, G., Wehrse, R., and Wagoner, R.V. 1985, Ap. J., 289, 198.

Uomoto, A., and Kirshner, R.P. 1985, Astr. Ap., 149, L7.

Wagoner, R.V. 1981, Ap. J. (Lett.), 250, L65.

Weiler, K.W., Sramek, R.A., Panagia, N., van der Hulst, J.M., and Salvati, M. 1986, Ap. J., 301, 790.

Wesselink, A.J. 1946a, B.A.N., 10, 83.

Wesselink, A.J. 1946b, B.A.N., 10, 251.

Wesselink, A.J. 1985, in Supernovae as Distance Indicators, Lecture Notes in Physics, ed. N. Bartel (Springer-Verlag, Berlin), 224, 166.

Wheeler, J.C., and Levreault, R. 1985, Ap. J. (Lett.), 294 , L17. 